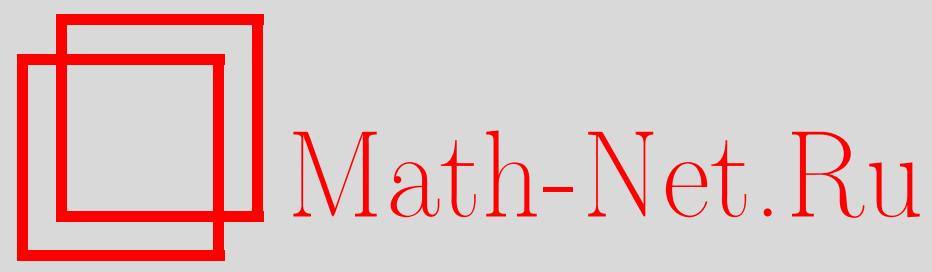

А. И. Кириллов, Обобщенные дифференцируемые продактмеры, Матем. заметки, 1998, том 63, выпуск 1, 37-55

DOI: https://doi.org/10.4213/mzm1246

Использование Общероссийского математического портала Math-Net.Ru подразумевает, что вы прочитали и согласны с пользовательским соглашением http://www.mathnet.ru/rus/agreement

Параметры загрузки:

IP: 54.205 .225 .156

26 апреля 2023 г., 16:16:21

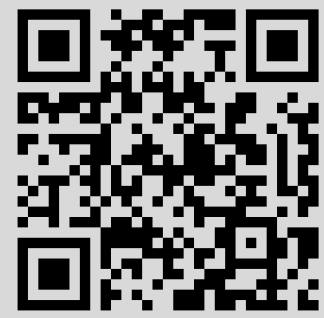




\section{ОБОБЩЕННЫЕ ДИФФЕРЕНЦИРУЕМЫЕ ПРОДАКТ-МЕРЫ}

\section{А. И. Кириллов}

Рассматривается класс мер на пространстве $\mathbb{R}^{\infty}$, определяемых последовательностями функций конечного числа переменных. Доказана теорема существования таких мер. Исследованы их свойства. Приведены конкретные примеры.

Библиограффия: 15 названий.

\section{$\S 1$. Введение}

1.1. Постановка задачи. Продакт-меры возникают естественньг образом, когда бывает задана последовательность пространств $X_{1}, X_{2}, \ldots$, снабженных $\sigma$-кольцами измеримых множеств $\mathfrak{M}_{1}, \mathfrak{M}_{2}, \ldots$, на которых определены вероятностные меры $\mu_{1}$, $\mu_{2}, \ldots$ В таком случае можно ввести пространство $X_{1} \times X_{2} \times \cdots$ и наименьшее $\sigma$-кольцо $\mathfrak{M}$, содержащееполукольцо $\mathfrak{M}_{0}$ множеств $\mathscr{M}_{1} \times \mathscr{M}_{2} \times \cdots$, у которых $\forall k \in \mathbb{N} \mathscr{M}_{k} \in \mathfrak{M}_{k}$ и только для конечного числа индексов $k \mathscr{M}_{k} \neq X_{k}$. На $\mathfrak{M}_{0}$ определена аддитивная функция множеств

$$
\mathscr{M}_{1} \times \mathscr{M}_{2} \times \cdots \rightarrow \mu_{0}\left(\mathscr{M}_{1} \times \mathscr{M}_{2} \times \cdots\right)=\prod_{k=1}^{\infty} \mu_{k}\left(\mathscr{M}_{k}\right)
$$

Если пространства $X_{k}$ польские и $\mathfrak{M}_{k}-\sigma$-кольца борелевских множеств, то существует единственное продолжение $\mu_{0}$ до $\sigma$-аддитивной меры $\mu$ на $\mathfrak{M}$ (см., например, [1, с. 123]). Мера $\mu$ называется продакт-мерой, ассоциированной $c$ семейством $\left\{X_{k}, \mathfrak{M}_{k}, \mu_{k}\right\}_{k=1}^{\infty}$.

Свойства продакт-меры $\mu$ выводятся из свойств мер $\mu_{k}$. Обычно пространства $X_{k}$ конечномерны и $\mu_{k}$ определяются плотностями $\varrho_{k}$ относительно соответствующих мер Лебега. В этом смысле продакт-меры определяются функциями и для их исследования можно применить методы конечномерного анализа. Поскольку других методов практически нет, среди всевозможных мер на функциональных пространствах лишш продакт-меры изучены достаточно подробно.

Класс продакт-мер широк. Однако, в бесконечномерном анализе нельзя обойтись только продакт-мерами или мерами, абсолютно непрерывными относительно продактмер. В частности, иллюстрации теорем примерами, основанными на продакт-мерах,

Работа выполнена при финансовой поддержке Российского фонда фундаментальных исследований, грант № 96-01-01701. 
нередко считаются тривиальньги. Поэтому интересны классы других мер, которые были бы столь же доступны анализу, как и продакт-меры, но не сводились бы к ним и имели нетривиальные применения. В данной работе описаны меры из одного такого класса, которые мы назьваем обобщенными продакт-мерами.

1.2. Определение обобщенной продакт-меры. Обозначим $\mathbb{N}$ множество натуральных чисел и предположим, что заданы две возрастающие последовательности $\{m(k)\}_{k=1}^{\infty},\{n(k)\}_{k=1}^{\infty} \subset \mathbb{N}$ такие, что $m(1)=1$ и $\forall k \in \mathbb{N} m(k+1) \leqslant n(k)+1$. Обозначим $N_{k}=[m(k), n(k)] \cap \mathbb{N}$. Очевидно, что $\bigcup_{k=1}^{\infty} \mathbb{N}_{k}=\mathbb{N}$ и каждое $n \in \mathbb{N}$ входит только в конечное число множеств $\mathbb{N}_{k}$. Пусть, как обычно, $\mathbb{R}^{\infty}$ - пространство последовательностей вещественных чисел и $\mathbb{R}_{0}^{\infty}$ - его подпространство, состоящее из последовательностей, у которых лишш конечное число членов отлично от нуля. Для любой последовательности $x=\left(x_{1}, x_{2}, \ldots\right) \in \mathbb{R}^{\infty}$ и $\forall k \in \mathbb{N}$ положим $\widehat{\Pi}_{k} x=\left(x_{m(k)}, \ldots, x_{n(k)}\right)$.

Допустим, что на каждом из пространств $\widehat{\Pi}_{k} \mathbb{R}^{\infty}$ можно определить неотрищательную функцию $\varrho_{k}$ таким образом, что выполняются следующие три условия:

1) $\forall q=\left(q_{1}, q_{2}, \ldots\right) \in \mathbb{R}_{0}^{\infty}$ существует

$$
\widetilde{\mu}(q)=\lim _{n \rightarrow \infty} \int_{\mathbb{R}^{M(n)}} \prod_{k=1}^{n} \varrho_{k}\left(x_{m(k)}, \ldots, x_{n(k)}\right) \prod_{j=1}^{M(n)} e^{i q_{j} x_{j}} d x_{j}
$$

где $M(n)=\max \{n(k): k \leqslant n\}-$ наибольшее число в $\bigcup_{k=1}^{n} \mathbb{N}_{k}$

2) $\widetilde{\mu}(0)=1$;

3) Сужение функции $\widetilde{\mu}(\cdot)$ на каждое конечномерное подпространство в $\mathbb{R}_{0}^{\infty}$ непрерывно.

Очевидно, что $\widetilde{\mu}(\cdot)$ удовлетворяет условиям теоремы Бохнера-Колмогорова (см., например, $[2, \S \mathrm{IV} .4 .2])$. Поэтому на цилиндрическом $\sigma$-кольце $\mathscr{C}\left(\left(\mathbb{R}_{0}^{\infty}\right)^{a}, \mathbb{R}_{0}^{\infty}\right)$ определена вероятностная мера $\mu_{a}$, для которой $\widetilde{\mu}(\cdot)$ является характеристическим функционалом. Пространство $\left(\mathbb{R}_{0}^{\infty}\right)^{a}$, алгебраически сопряженное к $\mathbb{R}_{0}^{\infty}$, каноническим образом отождествляется с $\mathbb{R}^{\infty}$. Обозначим через $\mu$ образ $\mu_{a}$ при таком отождествлении.

В случае, когда $\forall k \in \mathbb{N} m(k)=n(k)=k$, каждая функция $\varrho_{k}$ определяет вероятностную меру $\mu_{k}$ на $\mathscr{B}(\mathbb{R})$. Предел $(1)$ существует для любого $q \in \mathbb{R}_{0}^{\infty}$ и равен $\widetilde{\mu}_{1}\left(q_{1}\right) \cdots \widetilde{\mu}_{j}\left(q_{j}\right)$, где $j$ - номер последнего члена последовательности $q=\left(q_{1}, q_{2}, \ldots\right)$, отличного от нуля, и $\widetilde{\mu}_{k}(\cdot)(k=1, \ldots, j)$ - характеристические функции мер $\mu_{k}$. Непрерьвность $\widetilde{\mu}(\cdot)$ очевидна. Мера $\mu$ с характеристическим функционалом $\widetilde{\mu}(\cdot)$ является продакт-мерой, ассоциированной с семейством $\left\{X_{k}=\mathbb{R}, \mathfrak{M}_{k}=\mathscr{B}(\mathbb{R}), \mu_{k}\right\}_{k=1}^{\infty}$.

Вообще, предел (1) определяет характеристический функционал некоторой продактмеры, если множества из $\left\{\mathbb{N}_{k}\right\}_{k=1}^{\infty}$ не “сцеплены", т.е. не существует возрастающей последовательности натуральных чисел, каждый член которой принадлежит не менее чем двум множествам из семейства $\left\{\mathbb{N}_{k}\right\}_{k=1}^{\infty}$. Возможны и иные ситуации. Пусть, например, $N_{1}=\{1\}, N_{2}=\{1,2\}, \ldots, N_{k}=\{k-1, k\}, \ldots$ и

$$
\begin{gathered}
\varrho_{1}\left(x_{1}\right)=\pi^{-1}\left(1+x_{1}^{2}\right)^{-1}, \varrho_{2}\left(x_{1}, x_{2}\right)=\pi^{-1}\left(1+x_{1}^{2}\right)^{1 / 2}\left(1+x_{1}^{2}+x_{2}^{2}\right)^{-1}, \ldots, \\
\varrho_{k}\left(x_{k-1}, x_{k}\right)=\frac{1}{\pi} \frac{\sqrt{1+x_{k-1}^{2}}}{1+x_{k-1}^{2}+x_{k}^{2}}, \ldots
\end{gathered}
$$


В данном случае (1) определяет некоторую вероятностную меру на $\mathbb{R}^{\infty}$, которая не является продакт-мерой. Меры, определяемые характеристическими функционалами (1) для всевозможных семейств $\left\{\mathbb{N}_{k}\right\}_{k=1}^{\infty},\left\{\varrho_{k}\right\}_{k=1}^{\infty}$, назьваются в данной статье обобщенными продакт-мерами. Мы рассмотрим некоторые дифференцируемые меры такого класса.

1.3. Дифференцируемые меры. Напомним, что мера $\mu$ на $\sigma$-кольце $\mathfrak{M}$ измеримых подмножеств линейного пространства $X$ называется дифференцируемой по некоторому направлению $h \in X$, если $\forall A \in \mathfrak{M}$ существует

$$
\mu_{h}^{\prime}(A)=\lim _{t \rightarrow 0} \frac{\mu(A+t h)-\mu(A)}{t} .
$$

$\Phi$ ункция множеств $\mu_{h}^{\prime} \sigma$-аддитивна и абсолютно непрерывна относительно $\mu$ (см., например, [3]). По теореме Радона-Никодима существует функция $\beta_{h} \in L^{1}(X, \mu)$ такая, что $\forall A \in \mathfrak{M}$

$$
\mu_{h}^{\prime}(A)=\int_{A} \beta_{h}(x) d \mu(x)
$$

Функция $\beta_{h}$ назьвается логарифмической производной меры $\mu$ по направлению $h$.

Пусть $\mu$-продакт-мерана $\mathbb{R}^{\infty}$, определяемая последовательностью плотностей $\left\{\varrho_{k}:\right.$ $\mathbb{R} \rightarrow \mathbb{R}\}_{k=1}^{\infty}$, имеющих обобщенные производные. Тогда $\mu$ дифференцируема по направлению каждого вектора $e_{k}$ канонического базиса пространства $\mathbb{R}^{\infty}$. При этом $\forall k \in \mathbb{N}$

$$
\beta_{e_{k}}(x)=\frac{\partial}{\partial x_{k}} \ln \varrho_{k}\left(x_{k}\right) .
$$

Очевидно, что логарифмические производные продакт-меры по направлениям $e_{k}$ $(k \in \mathbb{N})$ являются функциями одной переменной. Обобщенные продакт-меры характерны тем, что их логарифмические производные по направлениям $e_{k}(k \in \mathbb{N})$ являются функциями конечного числа переменных. Точнее, если задана некоторая система $\left\{N_{k}\right\}_{k=1}^{\infty}$ и последовательность неотрицательных функций $\varrho_{k}: \widehat{\Pi}_{k} \mathbb{R}^{\infty} \rightarrow \mathbb{R}$, определяющих обобщенную дифференцируемую продакт-меру $\mu$, то логарифмические производные меры $\mu$ имеют вид

$$
\beta_{j}: x \in \mathbb{R}^{\infty} \mapsto \beta_{j}\left(x_{l(j)}, \ldots, x_{r(j)}\right)=\sum_{k=1}^{\infty} \frac{\partial}{\partial x_{j}} \ln \varrho_{k}\left(x_{m(k)}, \ldots, x_{n(k)}\right),
$$

где $l(j)$ - левый конец первого из множеств $\left\{N_{k}\right\}_{k=1}^{\infty}$, содержаших $j$, a $r(j)$ - правьй конец последнего из таких множеств.

1.4. Содержание статьи. В данной работе условия, при которьх функции $\left\{\varrho_{k}\right\}_{k=1}^{\infty}$ определяют обобщенную продакт-меру на $\mathbb{R}^{\infty}$, вьводятся из условий, при которых функции $\left\{\beta_{j}\right\}_{j=1}^{\infty}$ являются логарифмическими производными некоторой меры на $\mathbb{R}^{\infty}$. Такие условия были исследованы в работах [4]-[9]. Применительно к функциям вида (2) их можно ослабить. Кроме того, некоторые дополнительные упрощения обусловлены тем, что в данной работе искомые меры задаются на $\mathbb{R}^{\infty}$, тогда как в [4]-[9] меры строились на гильбертовом пространстве.

Статья организована следующим образом. В $\S 2$ мы установим критерий существования обобщеннњх продакт-мер и исследуем их дифференцируемость. В 33 мы приведем 
несколько примеров обобщенных продакт-мер. В частности, в $\S 3$ показано, что класс обобщенных продакт-мер включает в себя смеси гауссовых мер, рассмотренные в [10]. В $\S 3$ описано также применение обобшенных продакт-мер в статистической механике систем, состоящих из бесконечного числа частиц. (Мы имеем в виду применение обобщенных продакт-мер и в предельных теоремах для зависимых случайных величин, но об этом в данной статье речь идти не будет.) В $\S 4$ описаны свойства обобщенных продакт-мер.

Для прояснения сути дела в данной статье за исключением п. 3.2 и п. 3.6 мы ограничиваемся случаем, когда каждое $n \in \mathbb{N}$ принадлежит не более чем двум множествам из совокупности $\left\{N_{k}\right\}_{k=1}^{\infty}$, т.е. когда $\forall k \in \mathbb{N} n(k)<m(k+2)$. Обобщенные продакт-меры с более сложным зацеплением множеств $\left\{N_{k}\right\}_{k=1}^{\infty}$ трактуются аналогично.

\section{§ 2. Существование и дифференцируемость обобщенных продакт-мер}

2.1. Вспомогательная теорема. Пусть заданы множества

$$
\left\{N_{k}=[m(k), n(k)] \cap \mathbb{N}\right\}_{k=1}^{\infty}
$$

и неотрицательные функции $\left\{\varrho_{k}: \widehat{\Pi}_{k} \mathbb{R}^{\infty} \rightarrow \mathbb{R}\right\}_{k=1}^{\infty}$ такие, что $\forall k \in \mathbb{N}$ на пространстве $\mathbb{R}^{n(k)}$ определена вероятностная мера $\mu_{k}$ с плотностью

$$
x \in \mathbb{R}^{n(k)} \mapsto \prod_{j=1}^{k} \varrho_{j}\left(x_{m(j)}, \ldots, x_{n(j)}\right)
$$

Напомним, что $\forall j \in \mathbb{N} r(j)=\max \{n(k): m(k) \leqslant j \leqslant n(k)\}-$ правьй конец последнего из множеств $\left\{N_{k}\right\}_{k=1}^{\infty}$, содержащих $j$. Обозначим $V^{2,1}\left(\mathbb{R}^{n}\right)$ пространство функций из $L^{1}\left(\mathbb{R}^{n}\right) \cap L^{2}\left(\mathbb{R}^{n}\right)$, имеющих обобщенные производные класса $L^{1}\left(\mathbb{R}^{n}\right) \cap L^{2}\left(\mathbb{R}^{n}\right)$ по каждой переменной. Справедлива следующая теорема.

Tеорема 1. Пусть $\forall k \in \mathbb{N} \varrho_{k} \in V^{2,1}\left(\widehat{\Pi}_{k} \mathbb{R}^{\infty}\right) u \forall j \in \mathbb{N}$ существует ограниченное мнохсество $U_{j} \subset \mathbb{R}^{r(j)}$, вне которого для любого $k \in \mathbb{N}$ такого, ито $n(k) \geqslant j$, $u$ для некоторых $q, v_{1}, \ldots, v_{j}>0$ выполняется неравенство

$$
q \max _{i \leqslant j} v_{i}+\sum_{i=1}^{j} v_{i}\left[1+x_{i} \frac{\partial}{\partial x_{i}} \sum_{s=1}^{k} \ln \varrho_{s}(x)\right]<-A_{j}<0
$$

Пусть, кроме того,

$$
\sup _{k} \int_{\mathbb{R}^{n(k)} \cap Q_{j}}\left|\sum_{i=1}^{j} v_{i} x_{i} \frac{\partial}{\partial x_{i}} \sum_{s=1}^{k} \ln \varrho_{s}(x)\right| d \mu_{k}(x)<\infty
$$

где $Q_{j}=\left\{x \in \mathbb{R}^{\infty}:\left(x_{1}, \ldots, x_{r(j)}\right) \in U_{j} \backslash B_{j}\right\}$ и $B_{j}$ - некоторый иар с иентром $\boldsymbol{в}$ начале координат.

Tогда $\forall j \in \mathbb{N}$

$$
\sup _{k} \int_{\mathbb{R}^{n(k)}}\left(\sum_{i=1}^{j} x_{i}^{2}\right)^{q / 2} d \mu_{k}(x)<\infty
$$


ЗАмечаниЕ. Разумеется, при каждом $k \in \mathbb{N}$ интеграл в левой части неравенства (4) ограничен. Условие (4) означает, что все такие интегралы ограничены в совокупности. Это условие выполняется, например, в случае, когда функции $\ln \varrho_{k}$ имеют производные, которые локально ограничены вне некоторого шара. Внутри этого шара функции $\ln \varrho_{k}$ могут иметь особенности. Условие (3) - это слабейшее из неравенств коэрцитивности для отображения

$$
x \in \mathbb{R}^{r(j)} \mapsto\left\{v_{1} \frac{\partial}{\partial x_{1}} \sum_{s=1}^{k} \ln \varrho_{k}(x), \ldots, v_{j} \frac{\partial}{\partial x_{j}} \sum_{s=1}^{k} \ln \varrho_{k}(x)\right\} \in \mathbb{R}^{j}
$$

ДоКАЗАТЕЛЬСТВо ТЕОРЕмЫ 1 . Для меры $\mu_{k}$ справедлива формула интегрирования по частям, из которой следует, что $\forall k \in \mathbb{N}$ и $\forall f \in C^{(1)}\left(\mathbb{R}^{n(k)}\right)$

$$
\int_{\mathbb{R}^{n(k)}} L_{k} f(x) d \mu_{k}(x)=0
$$

где

$$
L_{k}=\sum_{i=1}^{n(k)} v_{i}\left(\frac{\partial^{2}}{\partial x_{i}^{2}}+\beta_{i}^{(k)} \frac{\partial}{\partial x_{i}}\right) \quad \text { и } \quad \beta_{i}^{(k)}(x)=\frac{\partial}{\partial x_{i}} \sum_{s=1}^{k} \ln \varrho_{s}(x) .
$$

Фиксируем произвольное $j \in \mathbb{N}$ и положим $\|x\|_{j}^{2}=\sum_{i=1}^{j} x_{i}^{2}$. Обозначим через $r$ радиус шара $B_{j}$. Выберем некоторую неубывающую функцию $f \in C_{\text {loc }}^{(2)}(\mathbb{R})$ такую, что $f(x)=0$ при $0 \leqslant x \leqslant r$ и $f(x)=x$ при $x>3 r / 2$. Положим

$$
V(x)=\frac{f\left(\|x\|_{j}^{q+2}\right)}{1+\alpha f\left(\|x\|_{j}^{q+2}\right)}, \quad \widetilde{U}_{j}=\left\{x \in \mathbb{R}^{\infty}:\left(x_{1}, \ldots, x_{r(j)}\right) \in U_{j} \cup\left(3 B_{j} / 2\right)\right\} .
$$

Нетрудно проверить, что если $n(k) \geqslant j$, то

$$
\begin{aligned}
L_{k} V(x) \leqslant & \frac{(q+2)\|x\|_{j}^{q} f^{\prime}\left(\|x\|_{j}^{q+2}\right)}{\left(1+\alpha f\left(\|x\|_{j}^{q+2}\right)\right)^{2}}\left[q \max _{i \leqslant j} v_{i}+\sum_{i=1}^{j} v_{i}\left(1+x_{i} \beta_{i}^{(k)}(x)\right)\right] \\
& +\frac{(q+2)^{2}\|x\|_{j}^{2 q+2} f^{\prime \prime}\left(\|x\|_{j}^{q+2}\right)}{\left(1+\alpha f\left(\|x\|_{j}^{q+2}\right)\right)^{2}} \max _{i \leqslant j} v_{i} .
\end{aligned}
$$

Пользуясь $(6),(7),(3)$ и (4), получаем, что

$$
\sup _{k} \int_{\mathbb{R}^{n(k)} \backslash U_{j}} \frac{\|x\|_{j}^{q}}{\left(1+\alpha\|x\|_{j}^{q+2}\right)^{2}} d \mu_{k}(x)<\infty .
$$

Отсюда в силу леммы $Ф$ ату следует (5). Теорема 1 доказана. 
2.2. Существование обобщенных продакт-мер. Для любых $k \in \mathbb{N}$ и $j=1, \ldots$, $n(k)$ обозначим через $\mu_{k}, j$ проекцию меры $\mu_{k}$ на пространство $\mathbb{R}^{j}$, состоящее из тех элементов пространства $\mathbb{R}^{n(k)}$, у которых все координаты с номерами больше $j$ равны 0 . Мы будем естественным образом отождествлять пространства $\mathbb{R}^{n}$ с подпространствами из $\mathbb{R}_{0}^{\infty}$. В этом смысле $\forall n_{1}, n_{2} \in \mathbb{N} n_{1} \leqslant n_{2} \Longrightarrow \mathbb{R}^{n_{1}} \subset \mathbb{R}^{n_{2}}$.

Из неравенства (5) следует, что $\forall j \in \mathbb{N}$

$$
\sup _{k} \int_{\mathbb{R}^{j}}\left(\sum_{i=1}^{j} x_{i}^{2}\right)^{q / 2} d \mu_{k, j}(x)<\infty .
$$

Поэтому $\forall j \in \mathbb{N}$ семейство мер $\left\{\mu_{k, j}: k \in \mathbb{N}, n(k) \geqslant j\right\}$ относительно компактно в слабой топологии пространства мер на $\mathbb{R}^{j}$. Значит, последовательность $\mu_{1}, \mu_{2}, \ldots$ содержит подпоследовательность различных мер, проекции которых на $\mathbb{R}^{1}$ слабо сходятся к некоторой вероятностной мере $\nu_{1}$ на $\mathbb{R}^{1}$. Обозначим эту подпоследовательность через $\mu_{k(1,1)}, \mu_{k(2,1)}, \ldots$. Из нее можно выбрать подпоследовательность различных мер, проекции которых на $\mathbb{R}^{2}$ слабо сходятся к некоторой вероятностной мере $\nu_{2}$ на $\mathbb{R}^{2}$. Эту подпоследовательность мы обозначим через $\mu_{k(1,2)}, \mu_{k(2,2)}, \ldots$ Продолжая такой процесс, мы получим бесконечную матрищу натуральных чисел $\{k(i, j)\}_{i, j=1}^{\infty}$ и последовательность мер $\nu_{1}, \nu_{2}, \ldots$ на $\mathbb{R}^{1}, \mathbb{R}^{2}, \ldots$, причем

$$
\nu_{n}=w-\lim _{i \rightarrow \infty} \mu_{k(i, n), n} .
$$

На пространстве $\mathbb{R}_{0}^{\infty}$ определим функцию $\widetilde{\mu}(\cdot): \mathbb{R}_{0}^{\infty} \rightarrow \mathbb{C}$, положив $\forall n \in \mathbb{N}, \forall q \in$ $\mathbb{R}^{n} \subset \mathbb{R}_{0}^{\infty}$

$$
\widetilde{\mu}(q):=\int_{\mathbb{R}^{n}} \exp \left(i \sum_{k=1}^{n} q_{k} x_{k}\right) d \nu_{n}(x)
$$

Легко проверить, что при $m \geqslant n$ проекция меры $\nu_{m}$ на $\mathbb{R}^{n}$ равна $\nu_{n}$. Поэтому $\widetilde{\mu}(\cdot)$ не зависит от числа $n$, фигурирующего в правой части (9).

Поскольку пространство, алгебраически сопряженное к $\mathbb{R}_{0}^{\infty}$, отождествимо с $\mathbb{R}^{\infty}$, из теоремы Бохнера-Колмогорова [2, IV.4.2] следует, что на $\mathbb{R}^{\infty}$ определена вероятностная мера $\mu$, характеристический функционал которой равен $\widetilde{\mu}(\cdot)$. Сопоставляя $(9)$ с (1) и учитьвая (8), мы видим, что $\mu$ есть обобщенная продакт-мера, ассоциированная с последовательностями $\left\{N_{j}^{\prime}\right\}_{j=1}^{\infty},\left\{\varrho_{j}^{\prime}\right\}_{j=1}^{\infty}$, где $\forall j \in \mathbb{N}$

$$
N_{j}^{\prime}=\bigcup_{k=k_{j}}^{k_{j+1}} N_{k}, \quad \varrho_{j}^{\prime}=\prod_{k=k_{j}}^{k_{j+1}} \varrho_{k}
$$

и $k_{j}=k(j, j)$. Мы будем назьвать $\left\{N_{j}^{\prime}\right\}_{j=1}^{\infty},\left\{\varrho_{j}^{\prime}\right\}_{j=1}^{\infty}$ модификацией $\left\{N_{j}\right\}_{j=1}^{\infty},\left\{\varrho_{j}\right\}_{j=1}^{\infty}$. Таким образом, доказана следующая теорема.

ТЕорема 2. При условиях теоремы 1 существует модификация $\left\{N_{j}\right\}_{j=1}^{\infty}$, $\left\{\varrho_{j}\right\}_{j=1}^{\infty}$, определяющая обобщенную продакт-меру на $\mathbb{R}^{\infty}$. 
2.3. Дифференцируемость обобщенных продакт-мер. Доказательство теоремы 2 показьвает, что существует бесконечное множество модификаций $\left\{N_{k}\right\}_{k=1}^{\infty}$, $\left\{\varrho_{k}\right\}_{k=1}^{\infty}$, определяющих обобщенные продакт-меры. В $\S 4$ мы рассмотрим некоторые условия, при которых все такие меры совпадают, а здесь мы исследуем их дифференцируемость. Справедлива следующая теорема.

ТЕОРема 3. Пусть семейства $\left\{N_{k}\right\}_{k=1}^{\infty},\left\{\varrho_{k}\right\}_{k=1}^{\infty}$ определяют обобщенную продакт-меру $\mu$ на $\mathbb{R}^{\infty}$, причем для некоторого $j \in \mathbb{N}$ и всех $k \in \mathbb{N}$ существуют обобщенные производные $\partial \varrho_{k} / \partial x_{j} \in L^{1}\left(\widehat{\Pi}_{k} \mathbb{R}^{\infty}\right) \cap L^{2}\left(\widehat{\Pi}_{k} \mathbb{R}^{\infty}\right)$ и справедливо неравенство

$$
\sup _{k} \int_{\mathbb{R}^{n(k)}} \beta_{j}^{(k)}(x)^{2} d \mu_{k}(x)<\infty
$$

Тогда мера $\mu$ дифферениируема по направлению $j$-й координатной оси пространства $\mathbb{R}^{\infty}$, и ее логарифмическая производная $\beta_{j}$ принадлежит $L^{2}\left(\mathbb{R}^{\infty}, \mu\right)$.

ДокАЗАТЕЛЬСтво. Пусть $m, k \in \mathbb{N}$ таковы, что $n(k) \geqslant m \geqslant j$. Для произвольной функции $\varphi \in C^{(1)}\left(\mathbb{R}^{m}\right)$ справедлива формула интегрирования по частям

$$
\int_{\mathbb{R}^{n(k)}} \frac{\partial \varphi}{\partial x_{j}} d \mu_{k}=-\int_{\mathbb{R}^{n(k)}} \varphi \beta_{j}^{(k)} d \mu_{k}
$$

Поэтому существует положительное число $Q$ такое, что

$$
\left|\int_{\mathbb{R}^{m}} \frac{\partial \varphi}{\partial x_{j}} d \mu_{k, m}\right| \leqslant Q\left\{\int_{\mathbb{R}^{m}} \varphi^{2} d \mu_{k, m}\right\}^{1 / 2} .
$$

Переходя к пределу при $k \rightarrow \infty$, получаем, что линейньй функционал

$$
\varphi \in C^{(1)}\left(\mathbb{R}^{m}\right) \rightarrow \int_{\mathbb{R}^{\infty}} \frac{\partial \varphi}{\partial x_{j}} d \mu
$$

равномерно по $m$ непрерывен в топологии пространства $L^{2}\left(\mathbb{R}^{\infty}, \mu\right)$. Поэтому существует функция $\beta_{j} \in L^{2}\left(\mathbb{R}^{\infty}, \mu\right)$ такая, что $\forall \varphi \in \bigcup_{m=j}^{\infty} C^{(1)}\left(\mathbb{R}^{m}\right)$ справедлива формула интегрирования по частям

$$
\int_{\mathbb{R}^{\infty}} \frac{\partial \varphi}{\partial x_{j}} d \mu=-\int_{\mathbb{R}^{\infty}} \varphi \beta_{j} d \mu
$$

из которой, в свою очередь, следует утверждение теоремы.

2.4. Логарифмические производные обобщенных продакт-мер. Установим условия, при которых справедлива формула (2).

Пусть $f_{1}, f_{2}, \ldots$ - последовательность неотрицательных функций из $L^{1}(\mathbb{R})$, причем $\forall k \in \mathbb{N}\left\|f_{k}\right\|_{L^{1}}=1$. На пространстве $\mathbb{R}^{\infty}$ определим последовательность вероятностных мер $\mu^{(1)}, \mu^{(2)}, \ldots$, для которых при каждом $q \in \mathbb{R}_{0}^{\infty}$

$$
\tilde{\mu}^{(k)}(q)=\int_{\mathbb{R}^{\infty}} \exp \left(i \sum_{s=1}^{\infty} q_{s} x_{s}\right) \prod_{s=1}^{k} \varrho_{s}(x) \prod_{s=1}^{r(k)} d x_{s} \prod_{s=r(k)+1}^{\infty} f_{s}\left(x_{s}\right) d x_{s} .
$$


Иными словами, каждая мера $\mu^{(k)}$ есть произведение меры $\mu_{k}$ на $\mathbb{R}^{r(k)}$ и продакт-меры

$$
d \lambda_{k}=\prod_{s=r(k)+1}^{\infty} f_{s}\left(x_{s}\right) d x_{s}
$$

Легко проверить, что последовательность мер $\left\{\mu^{(k)}\right\}_{k=1}^{\infty}$ слабо сходится к $\mu$. Поэтому, если выполняются условия теоремы 3, то $\forall m \in \mathbb{N}, \forall \varphi \in C^{(1)}\left(\mathbb{R}^{m}\right)$

$$
\int_{\mathbb{R}^{\infty}} \frac{\partial \varphi}{\partial x_{j}} d \mu=\lim _{n \rightarrow \infty} \int_{\mathbb{R}^{\infty}} \frac{\partial \varphi}{\partial x_{j}} d \mu^{(n)}=-\lim _{n \rightarrow \infty} \int_{\mathbb{R}^{\infty}} \varphi \beta_{j} d \mu^{(n)} .
$$

Остается выяснить, при каких условиях

$$
\lim _{n \rightarrow \infty} \int_{\mathbb{R}^{\infty}} \varphi \beta_{j} d \mu^{(n)}=\int_{\mathbb{R}^{\infty}} \varphi \beta_{j} d \mu
$$

Это равенство справедливо, если, например, функция $\beta_{j}$ непрерьвна и ограничена. Другие условия рассматривались в [5]-[9]. В случае обобщенных продакт-мер эти условия упрощаются, и можно сформулировать следующее утверждение.

ТЕОРемА 4. Пусть семейства $\left\{N_{k}\right\}_{k=1}^{\infty},\left\{\varrho_{k}\right\}_{k=1}^{\infty}$ определяют обобщенную продакт-меру н на $\mathbb{R}^{\infty}$, причем $\forall j, k \in \mathbb{N}$

1) $\varrho_{k} \in V^{2,1}\left(\widehat{\Pi}_{k} \mathbb{R}^{\infty}\right)$;

2) функиии

$$
\beta_{j}^{(k)}(x)=\sum_{s=1}^{k} \varrho_{s}(x)^{-1} \frac{\partial}{\partial x_{j}} \varrho_{s}(x)
$$

непрерывны почти всюду по мере Лебега на $\mathbb{R}^{r(j)}$

3) справедливы неравенства (10).

Тогда мера $\mu$ дифферениируема по всем направлениям из $\mathbb{R}_{0}^{\infty}$, ее логарифмические производные принадлежат $L^{2}\left(\mathbb{R}^{\infty}, \mu\right)$ и определяются формулой (2).

ДокАЗАТЕЛьСТво. В силу теоремы 3 требуется проверить лишш то, что для логарифмических производных $\beta_{j}$ меры $\mu$ справедлива формула (2).

Фиксируем произвольное $j \in \mathbb{N}$. Выберем $k \in \mathbb{N}$ такое, что $n(k) \geqslant r(j)$. Проекция $\nu_{n(k)}$ меры $\mu$ на $\mathbb{R}^{n(k)}$ дифференцируема по всем направлениям из $\mathbb{R}^{n(k)}$ и, следовательно, абсолютно непрерьвна относительно меры Лебега на $\mathbb{R}^{n(k)}[11]$. Поэтому функция $\beta_{j}^{(k)}$ непрерьвна $\nu_{n(k)}$-почти всюду в $\mathbb{R}^{n(k)}$. В силу $(10) \forall \varphi \in C\left(\mathbb{R}^{n(k)}\right)$ интегралы

$$
\left\{\int_{\mathbb{R}^{n(k)}} \varphi(x) \beta_{j}^{(k)}(x) d \mu_{s, n(k)}(x)\right\}_{s=n(k)}^{\infty}
$$

равномерно абсолютно непрерьвны. Используя теоремы 5.2 (iii) и 5.4 из [12], мы можем утверждать, что $\forall \varphi \in C\left(\mathbb{R}^{n(k)}\right)$

$$
\lim _{s \rightarrow \infty} \int_{\mathbb{R}^{n(k)}} \varphi \beta_{j}^{(k)} d \mu_{s, n(k)}=\int_{\mathbb{R}^{n(k)}} \varphi \beta_{j}^{(k)} d \nu_{n(k)} .
$$


С учетом этого получаем, что $\forall \varphi \in C^{(1)}\left(\mathbb{R}^{n(k)}\right)$

$$
\begin{aligned}
\int_{\mathbb{R}^{\infty}} \varphi \beta_{j} d \mu & =-\int_{\mathbb{R}^{\infty}} \frac{\partial \varphi}{\partial x_{j}} d \mu=-\int_{\mathbb{R}^{n(k)}} \frac{\partial \varphi}{\partial x_{j}} d \nu_{n(k)}=-\lim _{s \rightarrow \infty} \int_{\mathbb{R}^{n(k)}} \frac{\partial \varphi}{\partial x_{j}} d \mu_{s, n(k)} \\
& =\lim _{s \rightarrow \infty} \int_{\mathbb{R}^{n(k)}} \varphi \beta_{j}^{(k)} d \mu_{s, n(k)}(x)=\int_{\mathbb{R}^{n(k)}} \varphi \beta_{j}^{(k)} d \nu_{n(k)}=\int_{\mathbb{R}^{\infty}} \varphi \beta_{j}^{(k)} d \mu .
\end{aligned}
$$

Отсюда следует, что $\beta_{j}=\beta_{j}^{(k)}$. Теорема 4 доказана.

\section{§3. Примеры}

3.1. Стохастические ядра. Рассмотрим последовательность произвольных неотрицательных функций $\varrho_{1}\left(x_{1}\right), \varrho_{2}\left(x_{1}, x_{2}\right), \ldots$, которые, начиная со второй, являются стохастическими ядрами, т.е. при $k \geqslant 2$

$$
\int_{\mathbb{R}} \varrho_{k}\left(x_{k-1}, x_{k}\right) d x_{k}=1
$$

Нетрудно проверить, что функции

$$
D_{1}\left(x_{1}\right)=\varrho_{1}\left(x_{1}\right), \quad D_{k}\left(x_{1}, \ldots, x_{k}\right)=\varrho\left(x_{1}\right) \prod_{j=2}^{k} \varrho_{j}\left(x_{j-1}, x_{j}\right)
$$

образуют последовательность согласованных конечномерных распределений, т.е. $\forall k \in \mathbb{N}$

$$
\int_{\mathbb{R}^{n}} D_{k+n}\left(x_{1}, \ldots, x_{k}, x_{k+1}, \ldots, x_{k+n}\right) d x_{k+1} \cdots d x_{k+n}=D_{k}\left(x_{1}, \ldots, x_{k}\right) .
$$

Следовательно, на $\mathbb{R}^{\infty}$ определена единственная вероятностная мера, проекции которой на пространства $\mathbb{R}^{1}, \mathbb{R}^{2}, \ldots, \mathbb{R}^{k}, \ldots$ совпадают с функциями $D_{1}, D_{2}, \ldots, D_{k}, \ldots$ Очевидно, что эта мера является обобщенной продакт-мерой.

В п. 4.2 будет показано, что каждая обобщенная продакт-мера, определяемая последовательностью $\varrho_{1}\left(x_{1}\right), \varrho_{2}\left(x_{1}, x_{2}\right), \ldots$, может быть задана с помощью последовательности стохастических ядер.

3.2. Смеси гауссовых мер. Описанньй в п. 3.1 элементарный способ построения обобщенных продакт-мер может быть применен и при более сложных зацеплениях, когда последовательность функций имеет вид $\left\{\varrho_{k}\left(x_{1}, \ldots, x_{k}\right)\right\}_{k=1}^{\infty}$ и

$$
\int_{\mathbb{R}} \varrho_{k}\left(x_{1}, \ldots, x_{k}\right) d x_{k}=1 .
$$

Например, если $\varrho_{1}\left(x_{1}\right)=\pi^{-1}\left(1+x_{1}^{2}\right)^{-1}$ и $\forall k \geqslant 2$

$$
\varrho_{k}\left(x_{1}, \ldots, x_{k}\right)=\frac{\left(1+x_{1}^{2}+\cdots+x_{k-1}^{2}\right)^{k / 2}}{\left(1+x_{1}^{2}+\cdots+x_{k}^{2}\right)^{(k+1) / 2}} / \int_{\mathbb{R}} \frac{d x}{\left(1+x^{2}\right)^{(k+1) / 2}},
$$

то соответствующая обобщенная продакт-мера является мерой Коши на $\mathbb{R}^{\infty}$. Она относится к классу смесей гауссовых мер, рассмотренному в [10]. Нетрудно проверить, что другие меры этого класса тоже являются обобщенными продакт-мерами, определяемыми функциями $\varrho_{k}$, для которых выполняется условие (11). 
3.3. Пример. Рассмотрим последовательность функций

$$
\varrho_{k}\left(x_{k}, x_{k+1}\right)=c_{k}\left[1+\exp \left(x_{k}^{2}+x_{k+1}^{2}\right)\right]^{-1},
$$

где $c_{1}, c_{2}, \ldots$ - нормировочные множители.

Непосредственные выгисления показывают, что

$$
-4\|x\|_{j}^{2} \leqslant \sum_{i=1}^{j} x_{i} \beta_{i}^{(k)}(x) \leqslant-\|x\|_{j}^{2}
$$

Следовательно, условия (3) и (4) теоремы 1 могут быть вьполнены при любом $q \geqslant 1$ и $v_{1}=v_{2}=\cdots=v_{j}$. Поэтому некоторая модификация последовательности функций (12) определяет обобщенную продакт-меру $\mu$ на $\mathbb{R}^{\infty}$. По теореме 4 эта мера дифференцируема вдоль всех направлений из $\mathbb{R}_{0}^{\infty}$. При этом логарифмические производные меры $\mu$ равны

$$
\beta_{1}(x)=-2 x_{1} \frac{e^{x_{1}^{2}+x_{2}^{2}}}{1+e^{x_{1}^{2}+x_{2}^{2}}}
$$

и $\forall j \geqslant 2$

$$
\beta_{j}(x)=-2 x_{j}\left[\frac{e^{x_{j-1}^{2}+x_{j}^{2}}}{1+e^{x_{j-1}^{2}+x_{j}^{2}}}+\frac{e^{x_{j}^{2}+x_{j+1}^{2}}}{1+e^{x_{j}^{2}+x_{j+1}^{2}}}\right] .
$$

3.4. Пример. Рассмотрим функции

$$
\varrho_{k}\left(x_{k}, x_{k+1}\right)=c_{k}\left(x_{k}^{2}+x_{k+1}^{2}+1\right)^{-\gamma_{k}},
$$

где $c_{1}, c_{2}, \ldots$ - нормировочные множители, а $\gamma_{1}, \gamma_{2}, \ldots$ удовлетворяют условиям

$$
\gamma_{i+1}>\gamma_{i}, \quad \sup \frac{\gamma_{i+1}}{\gamma_{i}}<\infty, \quad 2 \gamma_{1}^{-1}+\sum_{i=2}^{\infty} \gamma_{i}^{-1}<2
$$

Непосредственные вычисления показьвают, что $\forall j, k \in \mathbb{N}, j \geqslant 2, n(k) \geqslant j$,

$$
\sum_{i=1}^{j} v_{i} x_{i} \beta_{i}^{(k)}(x)=-2 \sum_{i=2}^{j} \gamma_{i-1} \frac{v_{i-1} x_{i-1}^{2}+v_{i} x_{i}^{2}}{1+x_{i-1}^{2}+x_{i}^{2}}-2 \gamma_{j} \frac{v_{j} x_{j}^{2}}{1+x_{j}^{2}+x_{j+1}^{2}}
$$

Положим $v_{1}=\gamma_{1}^{-1}$ и при $i \geqslant 2 v_{i}=\gamma_{i-1}^{-1}$. Используя $(15)$, получаем

$$
\left|\sum_{i=1}^{j} v_{i} x_{i} \beta_{i}^{(k)}(x)\right| \leqslant 2\left(1+\sum_{i=2}^{j} \frac{\gamma_{i}}{\gamma_{i-1}}\right)
$$

и

$$
\sum_{i=1}^{j} v_{i} x_{i} \beta_{i}^{(k)}(x) \leqslant-2 \sum_{i=1}^{j-1} \frac{z_{i}^{2}}{1+z_{i}^{2}}
$$


где $z_{i}^{2}=x_{i}^{2}+x_{i+1}^{2}$. Легко проверить, что

$$
\sum_{i=1}^{j-1} z_{i}^{2}>r^{2} \Longrightarrow \sum_{i=1}^{j-1} \frac{z_{i}^{2}}{1+z_{i}^{2}} \geqslant \frac{r^{2}}{1+r^{2}}
$$

Отсюда и из (16) следует, что условия (3) и (4) теоремы 1 выполняются, если $q=1$,

$$
U_{j}=\left\{x \in \mathbb{R}^{j}: \sum_{i=1}^{j-1}\left(x_{i}^{2}+x_{i+1}^{2}\right) \leqslant d^{2}\right\}
$$

и $d$ таково, что при $r>d$ справедливо неравенство

$$
\frac{2 r^{2}}{1+r^{2}}>\sum_{i=1}^{j} \gamma_{i}^{-1}
$$

Из (14) вытекает, что это неравенство имеет решение.

Итак, условия (3) и (4) теоремы 1 вьполнены. Поэтому некоторая модификация последовательности функций (13) определяет обобщенную продакт-меру на $\mathbb{R}^{\infty}$. По теореме 4 эта мера дифференцируема вдоль всех направлений из $\mathbb{R}_{0}^{\infty}$. При этом логарифмические производные меры $\mu$ равны

$$
\beta_{1}(x)=-2 x_{1} \frac{\gamma_{1}}{1+x_{1}^{2}+x_{2}^{2}}
$$

и $\forall j \geqslant 2$

$$
\beta_{j}(x)=-2 x_{j}\left(\frac{\gamma_{j-1}}{1+x_{j-1}^{2}+x_{j}^{2}}+\frac{\gamma_{j}}{1+x_{j}^{2}+x_{j+1}^{2}}\right) .
$$

3.5. Пример. Рассмотрим функции

$$
\varrho_{k}(x)=\left|x_{k}\right|^{b_{k}} \varrho_{k}^{(0)}(x)
$$

где функции $\varrho_{k}^{(0)}(\cdot)$ определены с помощью $(12)$ и $b_{1}, b_{2}, \ldots$ - неотрицательные числа. В данном случае

$$
-4\|x\|_{j}^{2}+\sum_{i=1}^{j} v_{i} b_{i} \leqslant \sum_{i=1}^{j} v_{i} x_{i} \beta_{i}^{(k)}(x) \leqslant-\|x\|_{j}^{2}+\sum_{i=1}^{j} v_{i} b_{i} .
$$

Следовательно, доказательство существования обобщенной продакт-меры, порождаемой функциями (17), можно выполнить так же, как и в п. 3.3. Заметим, что если $\forall m \in \mathbb{N}$ $\exists i \in \mathbb{N}, i>m$ такое, что $b_{i}>0$, то эта мера не является абсолютно непрерывной относительно меры $\mu$, описанной в п. 3.3, поскольку $\prod_{i=1}^{\infty}\left|x_{i}\right|^{b_{i}}$ расходится $\mu$-почти всюду. 
3.6. Меры Гиббса. Обобщенные продакт-меры неявно фигурируют в качестве основных объектов в статистической механике систем, состоящих из бесконечного числа частиц. Простейшим примером такой системы является одномерная цепочка атомов. Ее энергия имеет вид

$$
E=\sum_{i=1}^{\infty} \Phi_{i}\left(x_{i}\right)+\sum_{i=1}^{\infty} \sum_{j=i+1}^{\infty} V_{i j}\left(x_{i}-x_{j}\right),
$$

где $\Phi_{i}$ - потенциалы взаимодействия атомов с внешними полями и $V_{i j}-$ потенциалы взаимодействия атомов между собой. Если силы взаимодействия атомов обращаются в 0 при расстоянии между атомами, большем некоторого фиксированного числа, то лишь конечное число членов ряда $\sum_{j=i+1}^{\infty} V_{i j}\left(x_{i}-x_{j}\right)$ отлично от 0, и мы можем представить (18) в виде

$$
E=\sum_{i=1}^{\infty} \Phi_{i}\left(x_{i}\right)+\sum_{i=1}^{\infty} \sum_{j=i+1}^{i+n} V_{i j}\left(x_{i}-x_{j}\right) .
$$

Согласно постулату Гиббса вероятностное распределение координат $\left(x_{1}, \ldots, x_{N}\right)$ цепочки из конечного числа атомов имеет плотность

$$
d_{N}\left(x_{1}, \ldots, x_{N}\right)=c_{N} \exp \left\{-\alpha\left[\sum_{i=1}^{N} \Phi_{i}\left(x_{i}\right)+\sum_{i=1}^{N} \sum_{j=i+1}^{N \wedge(i+n)} V_{i j}\left(x_{i}-x_{j}\right)\right]\right\}
$$

Для любого $k \in \mathbb{N}$ и $N>k$ определим

$$
d_{N, k}\left(x_{1}, \ldots, x_{N}\right)=\int d_{N}\left(x_{1}, \ldots, x_{k}, x_{k+1}, \ldots, x_{N}\right) d x_{k+1} \cdots d x_{N} .
$$

Основной задачей статистической механики является выяснение условий, при которых $\forall k \in \mathbb{N}$ на $\mathbb{R}^{k}$ существует вероятностная мера $\mu_{k}$, плотность которой относительно меры Лебега есть функция $D_{k}\left(x_{1}, \ldots, x_{k}\right)$, для которой в том или ином смысле

$$
D_{k}\left(x_{1}, \ldots, x_{k}\right)=\lim _{N \rightarrow \infty} d_{N, k}\left(x_{1}, \ldots, x_{k}\right) .
$$

Очевидно, что меры $\mu_{1}, \mu_{2}, \ldots$ являются проекциями обобщенной продакт-меры, ассоциированной с семейством функций

$$
\varrho_{k}(x)=c_{k} \exp \left\{-\alpha \Phi_{k}\left(x_{k}\right)-\alpha \sum_{j=k+1}^{k+n} V_{k j}\left(x_{k}-x_{j}\right)\right\}
$$

В случае, когда применимо приближение “взаимодействия ближайших соседей",

$$
\varrho_{k}(x)=c_{k} \exp \left\{-\alpha \Phi_{k}\left(x_{k}\right)-\alpha V_{k}\left(x_{k}-x_{k+1}\right)\right\} .
$$

При $\Phi_{k}=0$ можно ввести новые координаты $y_{2}=x_{2}-x_{1}, y_{3}=x_{3}-x_{2}, \ldots$ и с помощью функций $\varrho_{k}(y)=c_{k} \exp \left\{-\alpha V_{k}\left(y_{k}\right)\right\}$ определить на пространстве последовательностей $\left(y_{2}, y_{3}, \ldots\right)$ продакт-меру, характеризующую вероятностное распределение расстояний между соседними атомами цепочки. 
Если не все потенциалы $\Phi_{k}$ равны 0 , то положив $y_{1}=x_{1}, y_{2}=x_{2}-x_{1}, y_{3}=x_{3}-x_{2}$, $\ldots$, можно строить на $\mathbb{R}^{\infty}$ обобщенную продакт-меру, ассоциированную с функциями

$$
\varrho_{k}(y)=c_{k} \exp \left\{-\alpha \Phi_{k}\left(y_{1}+\cdots+y_{k}\right)-\alpha V_{k}\left(y_{k}\right)\right\} \text {. }
$$

Другой подход состоит в том, чтобы строить обобщенную продакт-меру непосредственно с помощью функций (20). Преимуществом такого подхода является то, что функции (20) имеют более простое зацепление.

Заметим, что вопрос о единственности обобшенной продакт-меры, ассоциированной с заданным семейством функций типа (19) или (20), эквивалентен весьма важному вопросу о существовании фазовьх переходов в соответствующей системе частиц.

\section{§4. Некоторые свойства обобщенных продакт-мер}

4.1. Структура конечномерных проекций. Пусть $\mu$-обобщенная продакт-мера на $\mathbb{R}^{\infty}$, определяемая последовательностью функций $\left\{\varrho_{k}\left(x_{k}, x_{k+1}\right)\right\}_{k=1}^{\infty}$. Обозначим $\left\{k_{j}\right\}_{j=1}^{\infty}$ последовательность натуральных чисел такую, что $\forall n \in \mathbb{N}, f \in C\left(\mathbb{R}^{n}\right)$

$$
\begin{aligned}
& \lim _{j \rightarrow \infty} \int_{\mathbb{R}^{k_{j}+1}} f\left(x_{1}, \ldots, x_{n}\right) \varrho_{1}\left(x_{1}, x_{2}\right) \cdots \varrho_{k_{j}}\left(x_{k_{j}}, x_{k_{j}+1}\right) d x_{1} \cdots d x_{k_{j}+1} \\
& \quad=\int_{\mathbb{R}^{\infty}} f\left(x_{1}, \ldots, x_{n}\right) d \mu(x) .
\end{aligned}
$$

Положим $\forall n, m \in \mathbb{N}, m \geqslant n$,

$$
F_{n, m}(x)=\int_{\mathbb{R}^{m-n+1}} \varrho_{n}\left(x, x_{n+1}\right) \cdots \varrho_{m}\left(x_{m}, x_{m+1}\right) d x_{n+1} \cdots d x_{m+1} .
$$

Справедлива следующая теорема.

ТеОРема 5. Пусть выполнены условия:

1) мера $\mu$ на $\mathbb{R}^{\infty}$ определяется равенствами (21);

2) $\forall n \in \mathbb{N}$ и всех достаточно больиих $m \in \mathbb{N} \quad F_{n, m}(\cdot) \in L^{1}(\mathbb{R}) \cap L^{2}(\mathbb{R})$;

3) $\forall n \in \mathbb{N}$ и $p=1,2$ функиия

$$
x \mapsto \int_{\mathbb{R}^{n}} \varrho_{1}^{p}\left(x_{1}, x_{2}\right) \cdots \varrho_{n}^{p}\left(x_{n}, x\right) d x_{1} \cdots d x_{n}
$$

непрерывна, ограничена и при $p=1$ принадлежит $L^{2}(\mathbb{R}) ;$

4) $\forall n \in \mathbb{N}$ функиия

$$
g_{n}(\cdot)=\left[\int_{\mathbb{R}} \varrho_{n}\left(x_{n}, \cdot\right)^{2} d x_{n}\right]^{1 / 2}
$$

непрерывна и ограничена;

5) $\forall n \in \mathbb{N}$ существует полохстельное число q такое, что

$$
\sup _{m}\left\{\int_{\mathbb{R}}|x|^{q} F_{n, m}(x) d x / \int_{\mathbb{R}} F_{n, m}(x) d x\right\}<\infty .
$$


Тогда

1) $\forall n \in \mathbb{N}$ проекция меры $\mu$ на $\mathbb{R}^{n}$ абсолютно непрерывна относительно меры Лебега на $\mathbb{R}^{n}$ и соответствующая плотность имеет вид

$$
D_{n}\left(x_{1}, \ldots, x_{n}\right)=\varrho_{1}\left(x_{1}, x_{2}\right) \cdots \varrho_{n-1}\left(x_{n-1}, x_{n}\right) F_{n}\left(x_{n}\right) ;
$$

2) $\forall n \in \mathbb{N} \quad F_{n}(\cdot) \in L^{1}(\mathbb{R}) \cap L^{2}(\mathbb{R})$;

3) $\forall n \in \mathbb{N}$ последовательность $\left\{F_{n, m}\right\}_{m=n}^{\infty}$ содержит подпоследовательность, слабо сходящуюся $\kappa F_{n}$ в $L^{2}(\mathbb{R})$.

ЗАмечаниЕ. Аналогично тому, как была доказана теорема 1 , можно показать, что неравенство (22) вытекает из следующего условия: $\forall k \in \mathbb{N} \varrho_{k} \in V^{2,1}\left(\widehat{\Pi}_{k} \mathbb{R}^{\infty}\right)$ и существует ограниченное множество $U_{k} \subset \widehat{\Pi}_{k} \mathbb{R}^{\infty}$, вне которого справедливо неравенство

$$
q+1+x_{k} \frac{\partial}{\partial x_{k}} \ln \varrho_{k}\left(x_{k}, x_{k+1}\right)<-A_{k}<0
$$

и, кроме того,

$$
\sup _{m} \int_{W_{k, m}}\left|x_{k} \frac{\partial}{\partial x_{k}} \ln \varrho_{k}\left(x_{k}, x_{k+1}\right)\right| d \nu_{k, m}(x)<\infty,
$$

где $W_{k, m}=\left\{\left(x_{k}, \ldots, x_{k+m+1}\right):\left(x_{k}, x_{k+1}\right) \in U_{k} \backslash B_{k}\right\}, B_{k}$ - некоторый шар с центром в начале координат и $\nu_{k, m}-$ вероятностная мера с плотностью

$$
d_{k, m}\left(x_{k}, \ldots, x_{k+m+1}\right)=c_{k, m} \varrho_{k}\left(x_{k}, x_{k+1}\right) \cdots \varrho_{k+m}\left(x_{k+m}, x_{k+m+1}\right) .
$$

ДоКАЗАТЕЛЬСТво ТЕОРЕМЫ 5. Рассмотрим последовательность вероятностных мер на $\mathbb{R}$ с плотностями $c_{1, k_{j}} F_{1, k_{j}}(x)$, где индексы $j \in \mathbb{N}$ таковы, что $k_{j}>1$. Из условия (22) следует, что эти меры образуют относительно компактное множество. Следовательно, из указанной последовательности можно выделить подпоследовательность, слабо сходящуюся к некоторой вероятностной мере $\nu_{1}$ на $\mathbb{R}$. Обозначим через $J_{1}$ множество индексов $j$, соответствующих этой сходящейся подпоследовательности, и $j(1,1)$, $j(1,2), j(1,3), \ldots$ - элементы из $J_{1}$. Затем рассмотрим последовательность вероятностных мер на $\mathbb{R}$ с плотностями $c_{2, k_{j}} F_{2, k_{j}}(x)$ с индексами $j \in J_{1}$ такими, что $k_{j}>2$. В силу (22) из нее можно выделить подпоследовательность, слабо сходящуюся к некоторой вероятностной мере $\nu_{2}$ на $\mathbb{R}$. Пусть $J_{2} \subset J_{1}$ - множество индексов $j$, соответствующих этой подпоследовательности, и $j(2,1), j(2,2), \ldots$ - элементы из $J_{2}$. Продолжая этот процесс, мы получим бесконечную матрицу целых чисел $\{j(n, i)\}$. Обозначим $k(n, i)=k_{j(n, i)}$.

Имеем

$$
\lim _{i \rightarrow \infty} \int_{\mathbb{R}^{k(n, i)+1}} \varrho_{1}\left(x_{1}, x_{2}\right) \cdots \varrho_{k(n, i)}\left(x_{k(n, i)}, x_{k(n, i)+1}\right) d x_{1} \cdots d x_{k(n, i)+1}=1
$$

и $\forall n \in \mathbb{N}$ существует предел

$$
\begin{gathered}
\lim _{i \rightarrow \infty} c_{n, k(n, i)} \int_{\mathbb{R}^{n}} \varrho_{1}\left(x_{1}, x_{2}\right) \cdots \varrho_{n-1}\left(x_{n-1}, x_{n}\right) F_{n, k(n, i)}\left(x_{n}\right) d x_{1} \cdots d x_{n} \\
\quad=\lim _{i \rightarrow \infty} c_{n, k(n, i)} \int_{\mathbb{R}^{k(n, i)+1}} \prod_{s=1}^{k(n, i)+1} \varrho_{s-1}\left(x_{s-1}, x_{s}\right) d x_{s},
\end{gathered}
$$


где $\varrho_{0}(\cdot, \cdot)=1$. Следовательно, $\forall n \in \mathbb{N}$ существует

$$
c_{n}=\lim _{i \rightarrow \infty} c_{n, k(n, i)}>0 .
$$

Докажем теперь, что $\forall n \in \mathbb{N}$ проекция $\mu_{n}$ меры $\mu$ на пространство $\mathbb{R}^{n}$ абсолютно непрерьвна относительно меры Лебега. Пусть $f \in C\left(\mathbb{R}^{n}\right) \cap L^{2}\left(\mathbb{R}^{n}\right)$. Тог да

$$
\begin{aligned}
c_{n+1}\left|\int_{\mathbb{R}^{n}} f(x) d \mu_{n}(x)\right| \leqslant & \lim _{i \rightarrow \infty} c_{n+1, k(n+1, i)} \int_{\mathbb{R}^{n+1}}\left|f\left(x_{1}, \ldots, x_{n}\right)\right| F_{n+1, k(n+1, i)}\left(x_{n+1}\right) \\
& \times \prod_{s=1}^{n+1} \varrho_{s-1}\left(x_{s-1}, x_{s}\right) d x_{s} \\
\leqslant & \|f\|_{L^{2}\left(\mathbb{R}^{n}\right)} \lim _{i \rightarrow \infty} c_{n+1, k(n+1, i)} \int_{\mathbb{R}} F_{n+1, k(n+1, i)}(x) h(x) d x,
\end{aligned}
$$

где

$$
h(x)=\left[\int_{\mathbb{R}^{n}} \varrho_{1}\left(x_{1}, x_{2}\right)^{2} \cdots \varrho_{n}\left(x_{n}, x\right)^{2} d x_{1} \cdots d x_{n}\right]^{1 / 2}
$$

Поэтому

$$
\left|\int_{\mathbb{R}^{n}} f(x) d \mu_{n}\right| \leqslant O(1)\|f\|_{L^{2}\left(\mathbb{R}^{n}\right)},
$$

откуда следует, что мера $\mu_{n}$ абсолютно непрерывна относительно меры Лебега.

Пусть $f \in C(\mathbb{R}) \cap L^{2}(\mathbb{R})$. Тогда $\forall n \in \mathbb{N}$

$$
\begin{aligned}
&\left|\int_{\mathbb{R}} f(x) d \nu_{n}(x)\right| \leqslant \lim _{i \rightarrow \infty} c_{n, k(n+1, i)} \int_{\mathbb{R}^{k(n+1, i)-n+2}}\left|f\left(x_{n}\right)\right| \\
& \times \prod_{s=n}^{n+k(n+1, i)} \varrho_{s}\left(x_{s}, x_{s+1}\right) d x_{s} d x_{n+k(n+1, i)+1} \\
& \leqslant\|f\|_{L^{2}(\mathbb{R})} \frac{c_{n}}{c_{n+1}} \lim _{i \rightarrow \infty} c_{n+1, k(n+1, i)} \int_{\mathbb{R}} g_{n}\left(x_{n+1}\right) F_{n+1, k(n+1, i)}\left(x_{n+1}\right) d x_{n+1},
\end{aligned}
$$

где мы воспользовались тем, что $\{k(n+1, i)\}_{i=1}^{\infty}$ есть подпоследовательность последовательности $\{k(n, i)\}_{i=1}^{\infty}$. Поэтому

$$
\left|\int_{\mathbb{R}} f(x) d \nu_{n}(x)\right| \leqslant O(1)\|f\|_{L^{2}(\mathbb{R})},
$$

откуда следует, что $\forall n \in \mathbb{N}$ мера $\nu_{n}$ абсолютно непрерьвна относительно меры Лебега. Пусть $F_{n}$ - плотность меры $\nu_{n}$, деленная на $c_{n}$. По построению $F_{n} \in L^{2}(\mathbb{R}) \cap L^{1}(\mathbb{R})$.

Докажем теперь третье утверждение теоремы.

Пусть $f \in C(\mathbb{R}) \cap L^{2}(\mathbb{R})$. Тогда

$$
\begin{aligned}
\int_{\mathbb{R}} f(x) F_{n}(x) d x & =\frac{1}{c_{n}} \int_{\mathbb{R}} f(x) d \nu_{n}(x)=\frac{1}{c_{n}} \lim _{i \rightarrow \infty} \int_{\mathbb{R}} f(x) F_{n, m(n, i)}(x) d x \\
& =\lim _{i \rightarrow \infty} \int_{\mathbb{R}} f(x) F_{n, m(n, i)}(x) d x .
\end{aligned}
$$


Кроме того,

$$
\int_{\mathbb{R}} F_{n, k(n+1, i)}(x)^{2} d x \leqslant\left[\int_{\mathbb{R}} g_{n}(x) F_{n+1, k(n+1, i)}(x) d x\right]^{2},
$$

откуда следует, что $L^{2}$-нормы членов последовательности $\left\{F_{n, k(n+1, i)}\right\}_{i=1}^{\infty}$ ограничены в совокупности. Таким образом, эта последовательность сходится к $F_{n}(x)$ слабо в $L^{2}(\mathbb{R})[13$, теорема V.1.3]. Утверждение 3) доказано.

Теперь докажем формулу (23). Фиксируем $n \in \mathbb{N}$. Для любой $f \in C\left(\mathbb{R}^{n}\right)$

$$
\begin{aligned}
\int_{\mathbb{R}^{n}} f(x) d \mu_{n} & =\lim _{i \rightarrow \infty} \int_{\mathbb{R}^{n}} f\left(x_{1}, \ldots, x_{n}\right) F_{n, k(n+1, i)}\left(x_{n}\right) \prod_{s=1}^{n} \varrho_{s-1}\left(x_{s-1}, x_{s}\right) d x_{s} \\
& =\int_{\mathbb{R}^{n}} f\left(x_{1}, \ldots, x_{n}\right) F_{n}\left(x_{n}\right) \prod_{s=1}^{n} \varrho_{s-1}\left(x_{s-1}, x_{s}\right) d x_{s},
\end{aligned}
$$

так как функция

$$
x_{n} \mapsto \int_{\mathbb{R}^{n-1}} f\left(x_{1}, \ldots, x_{n}\right) \prod_{s=1}^{n-1} \varrho_{s}\left(x_{s}, x_{s+1}\right) d x_{s}
$$

принадлежит $L^{2}(\mathbb{R})$. Теорема 5 доказана.

ЗАмечАниЕ 1 . Теорему 5 можно применять, начиная с некоторого номера $n$. При меньших номерах представление (23) получается последовательньм интегрированием с учетом того, что $\forall n \in \mathbb{N}$

$$
F_{n}(x)=\int_{\mathbb{R}} \varrho_{n}(x, y) F_{n+1}(y) d y .
$$

ЗАмЕчАниЕ 2. Аналоги теоремы 5 справедливы для обобщенных продакт-мер, определяемых функциями $\varrho_{k}$ с более сложными зацеплениями, чем в (21).

4.2. Построение обобщенных продакт-мер с помощью стохастических ядер. Рассмотрим сначала случай, когда $\forall n \in \mathbb{N}$ функция $F_{n}(x)$, фигурируюшая в $(23)$, положительна. Введем функции

$$
\widetilde{\varrho}_{1}\left(x_{1}\right)=F_{1}(x), \quad \widetilde{\varrho}_{k}\left(x_{k-1}, x_{k}\right)=F_{k-1}\left(x_{k-1}\right)^{-1} \varrho_{k-1}\left(x_{k-1}, x_{k}\right) F_{k}\left(x_{k}\right) .
$$

Тогда в силу (24) при $k \geqslant 2$

$$
\int_{\mathbb{R}} \widetilde{\varrho}_{k}\left(x_{k-1}, x_{k}\right) d x_{k}=1
$$

т.е. функции $\widetilde{\varrho}_{k}-$ стохастические ядра. Плотности (23) можно представить в виде

$$
D_{n}\left(x_{1}, \ldots, x_{n}\right)=\widetilde{\varrho}_{1}\left(x_{1}\right) \widetilde{\varrho}_{2}\left(x_{1}, x_{2}\right) \cdots \widetilde{\varrho}_{n}\left(x_{n-1}, x_{n}\right),
$$

как в примере 3.1 .

Допустим теперь, что в некоторой точке $x_{0} \in \mathbb{R} F_{n}\left(x_{0}\right)=0$. Из (24) следует, что тогда для почти всех $y \varrho_{n}\left(x_{0}, y\right) F_{n+1}(y)=0$. Если функция $\varrho_{n}(\cdot, \cdot)$ положительна, это невозможно. Следовательно, если обобщенная продакт-мера определяется положительными функциями $\left(\varrho_{k}\right)$, то эта мера может быть задана с помощью стохастических ядер (25).

Если функции $\left(F_{n}\right)$ могут обращаться в 0 , то мы сохраним определения $(25)$ при $F_{k-1}\left(x_{k-1}\right)>0$ и положим $\widetilde{\varrho}_{k}\left(x_{k-1}, x_{k}\right)=0$ при $F_{k-1}\left(x_{k-1}\right)=0$. Тогда представление (27) будет по-прежнему справедливо, но условие (26) примет вид

$$
\int_{\mathbb{R}} \widetilde{\varrho}_{k}\left(x_{k-1}, x_{k}\right) d x_{k}=1 \quad \text { при } \widetilde{\varrho}_{k-1}\left(x_{k-2}, x_{k-1}\right)>0, \quad k \geqslant 2 .
$$


4.3. О единственности обобщенной продакт-меры. Рассмотрим пример использования теоремы 5 для доказательства единственности обобщенной продакт-меры, определяемой последовательностью $\left\{\varrho_{k}\left(x_{k}, x_{k+1}\right)\right\}_{k=1}^{\infty}$. В случае, когда $\forall k \in \mathbb{N}$

$$
\varrho_{k}\left(x_{k}, x_{k+1}\right)=\varrho\left(x_{k}, x_{k+1}\right) \text {, }
$$

равенство (24) принимает вид

$$
F_{n}(\cdot)=P F_{n+1}(\cdot)
$$

где $P$ - интегральньй оператор с ядром $\varrho(\cdot, \cdot)$. Справедливо следующее утверждение.

ТЕОрема 6. Пусть функиии (28) удовлетворяют условиям теоремы 5, оператор (29) непрерывен в $L^{2}(\mathbb{R})$, имеет изолированное простое собственное значение 1 и остальной его спектр принадлежит $[-r, r]$, где $r<1$. Тогда существует не более одной вероятностной меры на $\mathbb{R}^{\infty}$, определяемой последовательностью функиий (28).

ДокАзАТЕльство. Для любой функции $f \in L^{2}(\mathbb{R})$ последовательность $\left\{P^{k} f\right\}_{k=1}^{\infty}$ сходится либо к нулю, либо к собственному вектору оператора $P$, соответствующему

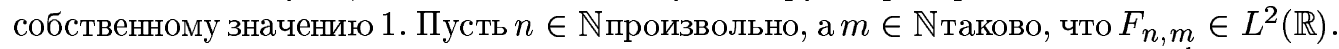
Тогда по теореме 5 некоторая подпоследовательность последовательности $\left\{P^{k} F_{n, m}\right\}_{k=1}^{\infty}$ слабо в $L^{2}(\mathbb{R})$ сходится к $F_{n}$. Следовательно, $F_{n}$ - собственная функция оператора $P$. Поэтому все обобщенные продакт-меры, определяемые различными модификациями последовательности (28), имеют одни и те же конечномерные проекции (23), т.е. совпадают. Теорема 6 доказана.

Для функций (12) можно принять

$$
\varrho(x, y)=c\left[1+\exp \left(x^{2}+y^{2}\right)\right]^{-1} .
$$

Соответственно, $P$ - симметричный, вполне непрерывный оператор с неразложимым ядром. Поэтому $P$ имеет единственное так назьваемое позитивное собственное значение [14]. Обозначим его через $\Lambda$. Для любого другого собственного значения $\lambda$ справедливо неравенство $\lambda<\Lambda$. Полагая в (30) $c=\Lambda^{-1}$, нетрудно проверить, что для соответствующих функций (28) выполняются условия теоремы 6. Поэтому обобщенная продакт-мера, описанная в п. 3.3, является единственной, определяемой функциями (12). Аналогично можно проверить, что мера, определяемая функциями (17) при одинаковых $b_{k}$, единственна.

4.4. Марковская единственность. Пусть $X^{*} \subset H \subset X$-оснащение вещественного сепарабельного гильбертова пространства $H$ локально вьпуклыми топологическими векторньми пространствами $X^{*}$ и $X$, причем $X^{*}$ - пространство, топологически сопряженное к $X$ относительно формы двойственности $\langle\cdot, \cdot\rangle: X^{*} \times X \rightarrow \mathbb{R}$, сужение которой на $X^{*} \times H$ совпадает со скалярным произведением $(\cdot, \cdot)_{H}$ в $H$. Для некоторого пространства $K \subset X^{*}$, всюду плотного в $H$, определим пространство цилиндрических функций

$$
C_{\mathrm{cyl}}^{\infty}=\left\{f\left(l_{1}, \ldots, l_{m}\right): m \in \mathbb{N}, f \in C_{b}^{\infty}\left(\mathbb{R}^{m}\right), l_{1}, \ldots, l_{m} \in K\right\} .
$$

Рассмотрим билинейную форму

$$
\mathscr{E}(u, v)=\int_{X}\left(\nabla_{H} u, \nabla_{H} v\right)_{H} d \mu, \quad u, v \in C_{\mathrm{cyl}}^{\infty},
$$


где $\forall x \in X \nabla_{H} u(x)$ - такой элемент пространства $H$, что $\forall l \in K$

$$
\left\langle l, \nabla_{H} u(x)\right\rangle=\left(l, \nabla_{H} u(x)\right)_{H}=\frac{\partial u}{\partial l}(x) .
$$

Допустим, что $\mu$ дифференцируема по всем направлениям $l \in K$, причем $\beta_{l} \in L^{2}(X, \mu)$. Тогда форма (31) имеет замкнутое расширение $(\mathscr{E}, \mathscr{D}(\mathscr{E}))$, являющееся формой Дирихле (см., например, [8] и цитированную там литературу). Генератор формы $(\mathscr{E}, \mathscr{D}(\mathscr{E}))$ обозначим $(\mathscr{L}, \mathscr{D}(\mathscr{L}))$. Нетрудно проверить, что если $K$ содержит ортонормированньй базис $e_{1}, e_{2}, \ldots$ пространства $H$, то $\forall u \in C_{\text {суl }}^{\infty}$

$$
\mathscr{L} u=\sum_{n=1}^{\infty}\left(\frac{\partial}{\partial e_{n}}\left(\frac{\partial u}{\partial e_{n}}\right)+\beta_{e_{n}} \frac{\partial u}{\partial e_{n}}\right)
$$

Правая часть этого равенства содержит только конечное число членов и определяет на $C_{\text {суl }}^{\infty}$ линейный оператор $L$. Будем говорить, что $\left(L, C_{\text {су1 }}^{\infty}\right)$ обладает свойством марковской единственности, если $(\mathscr{L}, \mathscr{D}(\mathscr{L}))$ - единственньй оператор Дирихле, являющийся расширением $\left(L, C_{\text {су1 }}^{\infty}\right)$. Это свойство представляет большой интерес, поскольку из него следует единственность решения мартингальной проблемы для $L$ [15].

Пусть $\forall n \in \mathbb{N} \mathscr{B}_{n} \subset \mathscr{B}(X)$ - наименьшее $\sigma$-кольцо, относительно которого измеримы все функции $\left\langle e_{j}, \cdot\right\rangle$ с $j=1, \ldots, n$ и $E_{\mu}\left(\cdot \mid \mathscr{B}_{n}\right)$ - условное математическое ожидание относительно $\mathscr{B}_{n}$. В [15] доказано, что если

$$
\sup _{n \in \mathbb{N}} \sum_{j=1}^{n}\left\|\beta_{e_{j}}-E_{\mu}\left(\beta_{e_{j}} \mid \mathscr{B}_{n}\right)\right\|_{L^{2}(X, \mu)}^{2}<\infty,
$$

то оператор $\left(L, C_{\text {су1 }}^{\infty}\right)$ обладает свойством марковской единственности. Для продакт-мер $\beta_{e_{j}}=E_{\mu}\left(\beta_{e_{j}} \mid \mathscr{B}_{n}\right)$, поэтому оператор $L$ обладает свойством марковской единственности при любом выборе пространства $H$, оснащенного $\mathbb{R}_{0}^{\infty}$ и $\mathbb{R}^{\infty}$.

Если $\mu$-обобщенная продакт-мера, то условие (32) упрощается. Предположим, что $\mu$ дифференцируема по всем направлениям из $K=\mathbb{R}_{0}^{\infty}=X^{*}$ и $\forall f \in \mathbb{R}_{0}^{\infty} \beta_{f} \in L^{2}\left(\mathbb{R}^{\infty}, \mu\right)$. Возьмем в качестве $e_{1}, e_{2}, \ldots$ канонический базис пространства $\mathbb{R}^{\infty}$. Для любого $j \in \mathbb{N}$ $\beta_{e_{j}}$ зависит лишь от конечного числа переменных. Например, если, как в $\S 3, \beta_{e_{j}}(x)=$ $\beta_{e_{j}}\left(x_{1}, \ldots, x_{j+1}\right)$, то

$$
\sum_{j=1}^{n}\left\|\beta_{e_{j}}-E_{\mu}\left(\beta_{e_{j}} \mid \mathscr{B}_{n}\right)\right\|_{L^{2}(X, \mu)}^{2}=\left\|\beta_{e_{n}}-E_{\mu}\left(\beta_{e_{n}} \mid \mathscr{B}_{n}\right)\right\|_{L^{2}(X, \mu)}^{2}
$$

и вместо (32) получаем

$$
\sup _{n}\left\|\beta_{e_{n}}-E_{\mu}\left(\beta_{e_{n}} \mid \mathscr{B}_{n}\right)\right\|_{L^{2}(X, \mu)}^{2}<\infty
$$

Поскольку

$$
\left\|\beta_{e_{n}}-E_{\mu}\left(\beta_{e_{n}} \mid \mathscr{B}_{n}\right)\right\|_{L^{2}(X, \mu)}^{2} \leqslant 4\left\|\beta_{e_{n}}\right\|_{L^{2}(X, \mu)}^{2},
$$

всегда можно найти гильбертово пространство $H$ такое, что $\mathbb{R}_{0}^{\infty}$ и $\mathbb{R}^{\infty}$ оснащают $H$ и соответствующий оператор $L$ обладает свойством марковской единственности. 
Для обобщенной продакт-меры, рассмотренной в п. 3.3,

$$
\left\|\beta_{e_{n}}-E_{\mu}\left(\beta_{e_{n}} \mid \mathscr{B}_{n}\right)\right\|_{L^{2}(X, \mu)}^{2} \leqslant C\left\|x_{n}\right\|_{L^{2}(X, \mu)}^{2} .
$$

Применяя к функции $v(x)=x_{n}^{4} /\left(1+\alpha x_{n}^{4}\right)$ те же преобразования, что и при доказательстве теоремы 1 , нетрудно проверить, что для любой обобщенной продакт-меры, ассоциированной с функциями $(12), \sup _{n}\left\|x_{n}\right\|_{L^{2}(X, \mu)}^{2}<\infty$. Следовательно, для каждой такой меры оператор $L$ обладает свойством марковской единственности при $H=\ell^{2}$.

Для обобщенной продакт-меры, рассмотренной в п. $3.4,\left|\beta_{e_{n}}\right| \leqslant 4 \gamma_{n+1}$. Поэтому оператор $L$ будет обладать свойством марковской единственности, если в качестве $H$ взять гильбертово пространство $\left\{x \in \mathbb{R}^{\infty}: \sum \gamma_{n+1}^{2} x_{n}^{2}<\infty\right\}$.

В случае, рассмотренном в п. $3.5, \beta_{e_{n}}(x)=b_{n} / x_{n}+\widetilde{\beta}_{e_{n}}(x)$, где $\widetilde{\beta}_{e_{n}}$ обозначает логарифмическую производную меры из п. 3.3. Поскольку

$$
\left\|\beta_{e_{n}}-E_{\mu}\left(\beta_{e_{n}} \mid \mathscr{B}_{n}\right)\right\|_{L^{2}(X, \mu)}^{2}=\left\|\widetilde{\beta}_{e_{n}}-E_{\mu}\left(\widetilde{\beta}_{e_{n}} \mid \mathscr{B}_{n}\right)\right\|_{L^{2}(X, \mu)}^{2},
$$

мы можем утверждать, что оператор $L$, ассоциированньй с обобщенной продакт-мерой из п. 3.5, обладает свойством марковской единственности при $H=\ell^{2}$.

В заключение автор выражает свою глубокую благодарность О. В. Зиминой за постоянное внимание, помощь и поддержку.

\section{СПИСОК ЦИТИРОВАННОЙ ЛИТЕРАТУРЫ}

[1] Неве ЖК. Математические основы теории вероятностей. М.: Мир, 1969.

[2] Вахания Н.Н., Тариеладзе В. И., Чобанян С. А. Вероятностные распределения в банаховых пространствах. М.: Наука, 1985.

[3] Богачёв В.И., Смолянов О.Г. Аналитические свойства бесконечномерных распределений // УМН. 1990. Т. 45. № 3. С. 3-83.

[4] Кириллов А. И. О двух математических проблемах канонического квантования. III. Стохастическая механика вакуума // ТМФ. 1992. Т. 91. № 3. С. 377-395.

[5] Кириллов А.И. О двух математических проблемах канонического квантования. IV // ТМФ. 1992. Т. 93. № 2. С. 249-263.

[6] Кириллов А.И. О задании мер на функциональных пространствах с помощью числовых плотностей и континуальных интегралов // Матем. заметки. 1993. Т. 53. № 5. С. 152-155.

[7] Кириллов А. И. Броуновское движение со сносом в гильбертовом пространстве и его применение в теории интегрирования // Теория вероятн. и ее применения. 1993. Т. 38. № 3. C. $629-634$.

[8] Кириллов А.И. Бесконечномерный анализ и квантовая теория как исчисления семимартингалов // УМН. 1994. Т. 49. № 3. С. 43-92.

[9] Кириллов А. И. О восстановлении мер по их логарифмическим производньм // Изв. РАН. Сер. матем. 1995. Т. 59. №1. С. 121-138.

[10] Норин Н. В., Смолянов О. Г. Несколько результатов о логарифмических производных мер на локально выпуклом пространстве // Матем. заметки. 1993. Т. 54. № 6. С. 135-138.

[11] Авербух В.И., Смолянов О.Г., Фомин С. В. Обобщенные функции и дифференциальные уравнения в линейных пространствах. І. Дифференцируемые меры // Тр. ММО. 1971. Т. 24. № 133-174.

[12] Биллингсли П. Сходимость вероятностных мер. М.: Наука, 1977.

[13] Йосида К. Функциональный анализ. М.: Мир, 1967.

[14] Красносельский М. А. Положительные решения операторных уравнений. М.: Физматгиз, 1962.

[15] Albeverio S., Röckner M., Zhang T. S. Markov uniqueness for a class of infinite-dimensional Dirichlet operators. Preprint SISSA № 223/92/FM, 1992. 